\title{
Beneficio de la realización de un taller en gestión emocional para enfermería nefrológica
}

\author{
“Accésit premio Janssen-Cilag de investigación en enfermería nefrológica 2011"
}

García-Llana Helena르, Barbero Gutiérrez Javier², Remor Bitencurt Eduardo³, Celadilla Díez Olga ${ }^{4}$, Trocoli González Filo ${ }^{4}$, Del Peso Gilsanz Gloria ${ }^{5}$, Selgas Gutiérrez Rafael ${ }^{5}$

${ }^{1}$ Psicóloga Investigadora. $S^{\circ}$ de Nefrología. Hospital Universitario La Paz-IdiPAZ

${ }^{2}$ Psicólogo Adjunto. ${ }^{\circ}$ de Hematología. Hospital Universitario La Paz

${ }_{3}^{3}$ Profesor Titular. Departamento de Psicología Biológica y de la Salud. Facultad de Psicología. Universidad

Autónoma de Madrid

${ }^{4}$ Enfermeras. Servicio de Nefrología. Hospital universitario La Paz, Madrid

${ }^{5}$ Nefrólogos. Servicio de Nefrología. Hospital Universitario La Paz, Madrid

\section{Resumen}

Los profesionales de enfermería del servicio de nefrología recibieron entrenamiento en gestión emocional mediante un taller de 12 horas centrado en la tecnología relacional de Counselling de amplia aplicabilidad en entornos sanitarios. El objetivo fue evaluar cambios en actitudes en relación a los principios bioéticos y en conocimientos sobre comunicación y gestión emocional antes y después de la implantación del taller. La muestra estaba formada por 63 profesionales del ámbito de la enfermería nefrológica (43 enfermeras y 20 auxiliares de enfermería) para la variable conocimientos y por 23 profesionales para la variable de actitudes (18 enfermeras y 5 auxiliares). Los resultados muestran cambios en implicación con los principios bioéticos $(p<0,05)$ y conocimientos $(p<0,001)$ en la muestra.

Correspondencia:

Helena García-Llana

Servicio de Nefrología. Hospital Universitario La Paz

Paseo de La Castellana, 261

28046, Madrid

e-mail: helenagllana@hotmail.com

PALABRAS CLAVE:

- FORMACIÓN

- GESTIÓN EMOCIONAL

- COMUNICACIÓN DIFÍCIL

- ENFERMERÍA

\section{Benefit of an emotional management workshop} for nephrology nursing staff

\section{Abstract}

Nursing professionals in the nephrology service received emotional management training in the form of a 12-hour workshop focused on the relational technology of counselling, which can be widely applied in healthcare environments. The aim was to assess changes in attitudes in relation to bioethical principles and in knowledge of communication and emotional management before and after the implementation of the workshop. The sample was made up of 63 professionals from the nephrology nursing area (43 nurses and 20 auxiliaries) for the knowledge variable and of 23 professionals for the attitudes variable (18 nurses and 5 auxiliaries). The results show changes in engagement with bioethical principles $(p<0.05)$ and knowledge $(p<0.001)$ in the sample. 


\section{KEY WORDS:}

- TRAINING

- EMOTIONAL MANAGEMENT

DIFFICULT COMMUNICATION

- NURSING

\section{Introducción}

El manejo adecuado de la tecnología médico-biológica, siendo imprescindible, resulta insuficiente si lo que pretendemos es dar una respuesta eficaz, efectiva y eficiente a la probable experiencia de sufrimiento que afronta el enfermo renal y su familia. Como afirma Chochinov' ${ }^{1}$, el "cuidado centrado en la dignidad", donde se potencia la comunicación y el abordaje emocional, tiene una importante influencia en la experiencia de ser paciente. La formación dentro de este marco de trabajo se puede aplicar, tanto a la práctica clínica, como a la formación de pre y postgrado de cualquier profesión sanitaria.

La dificultad de comunicación en situaciones de alta intensidad emocional y la falta de recursos a nivel profesional son factores que tienen un impacto nada despreciable sobre el tipo de asistencia que se ofrece $e^{2-3}$.

La formación del personal sanitario en habilidades de comunicación es necesaria para mejorar la calidad de vida del paciente renal, facilitar la adhesión a los tratamientos y facilitar la adaptación a la enfermedad ${ }^{4-7}$. Asimismo los programas formativos son un apoyo fundamental para los profesionales de enfermería, pues mejorando el trato con el paciente y la familia muchas veces se consigue una prevención de estrés laboral asistencial ${ }^{8-10}$.

Generalmente, este tipo de habilidades no se desarrollan eficazmente debido a la ausencia de formación de los profesionales de enfermería implicados en el cuidado del paciente crónico y a la falta de conciencia de dicha necesidad de formación en los mismos ${ }^{11}$.

Es importante recordar que la comunicación terapéutica se aprende, es decir, no viene incorporada de forma innata. Son necesarios escenarios mutuos de aprendizaje en los que se cree un ambiente facilitador para detectar y reconducir ciertos automatismos comunicativos de los que no siempre se es consciente y que pueden ser iatrogénicos en la relación con el paciente renal y su familia.

Desde la Supervisión de enfermería de nefrología de este hospital se promovió y facilitó el desarrollo de un taller de formación a enfermeras y auxiliares del servicio con el objetivo de mejorar actitudes, habilidades y conocimientos dentro del ámbito de la comunicación terapéutica.

En un trabajo previo ${ }^{12}$ describimos el impacto del mismo formato de taller sobre la totalidad de los profesionales del Servicio, y en este trabajo hemos decidido centrarnos en la categoría profesional de enfermería, presentando los resultados de este sub-grupo. Por tanto, nos planteamos el siguiente objetivo de estudio: Evaluar los cambios observados en el personal de enfermería en las actitudes relacionadas con los principios bioéticos, y en los conocimientos sobre comunicación y gestión emocional, antes y después de la implantación de un taller de gestión emocional.

\section{Material y métodos}

\section{Sujetos}

En este estudio se empleó un diseño pretest-postest sin grupo de control. La muestra inicial está compuesta por un total de 63 profesionales de enfermería del servicio de nefrología de un hospital terciario. Por categoría profesional están representados, el 93\% (43 sujetos) de las enfermeras, el 95\% (20 sujetos) de los auxiliares. Atendiendo a la edad, el $35 \%$ de la muestra tiene de 25 a 35 años, el 38\% tiene de 36 a 50 años, el 24\% tiene más de 50 años y el $3 \%$ tiene menos de 25 . El $95 \%$ son mujeres. El 49\% tiene más de 10 años de experiencia trabajando con enfermos renales.

\section{Instrumentos}

La evaluación se llevó a cabo con un cuestionario elaborado ad-hoc en las siguientes áreas:

Actitudes respecto a los 4 principios bioéticos (no maleficencia, justicia, autonomía y beneficencia):

a) Importancia de los principios bioéticos en el trabajo en el hospital $(p=0,74)$ : Consta de 4 ítems en una escala de respuesta tipo Likert que va desde el 1 (nada importante) a 10 (completamente importante). 
b) Implicación personal con los principios bioéticos en mi trabajo diario $(p=0,82)$ : Consta de 4 ítems en una escala de respuesta tipo Likert que va desde el 1 (nada implicado) a 10 (totalmente implicado).

Conocimientos ( $p=0,92)$ : Consta de 15 ítems con 4 opciones de respuesta $(1=$ acierto, $0=$ error $)$. Los fallos no penalizan. Se elaboraron dos categorías en el área de conocimientos: comunicación difícil y gestión emocional.

\section{Contenidos del taller}

Los contenidos de taller aparecen recogidos en la (Tabla 1).

\section{Sesión 1}

1. Detección de situaciones problemáticas en la práctica clínica diaria: se pide a los participantes un trabajo personal de cinco minutos escribiendo lo que cada uno percibe como más problemático desde el punto de vista emocional en su práctica clínica concreta. Posteriormente, se comparte con el grupo aprovechando la presentación personal que se pide a los alumnos.

2. El Counselling como herramienta para la comunicación terapéutica: se plantea un modelo de Counselling centrado en el entrenamiento acerca de actitudes, habilidades comunicativas y gestión emocional como herramientas de ayuda para la toma de decisiones en el paciente renal.

3. Modelo de intervención frente al sufrimiento: se describe el balance entre la percepción de amenaza a la integridad biológica 0 biográfica, y la percepción de contar con recursos internos o externos para hacer frente a esa amenaza.

4. Prevención del Burnout: Habilidades de autorregulación: desarrollo de habilidades cognitivas, emocionales y conductuales de detección de factores estresantes y de intervención frente a los mismos.

\section{Sesión 2}

5. Habilidades básicas comunicativas: entrenamiento en mensaje yo, validación, escucha activa, refuerzo, preguntas abiertas y focalizadas, como hacer y recibir críticas y denegación de peticiones.

6. Comunicación Difícil: protocolo de malas noticias, preguntas difíciles y manejo de reacciones emocionales intensas.

7. Relación terapéutica y deliberación: se desarrollan los modelos de relación clínica: paternalista, informativo, interpretativo y deliberativo.

Tabla 1. Contenidos del curso de Counselling en el Servicio de Nefrología

El taller se propuso a través del instrumento terapéutico del Counselling. El Counselling es un instrumento terapéutico que ha mostrado ser muy útil en el ámbito sanitario ${ }^{13-14}$. Consiste en un proceso interactivo-relacional entre paciente y profesional que fomenta la adaptación psicológica a la enfermedad, reduce los estados emocionales adversos, promueve la autorregulación del profesional y favorece la motivación al cambio ${ }^{15}$.

\section{Procedimiento}

Se realizaron cinco ediciones de un taller de 12 horas a lo largo del año 2007. El mismo fue impartido por un equipo de cuatro psicólogos del propio hospital con experiencia en la formación en comunicación a profesionales sanitarios.

\section{Análisis estadístico}

Para el análisis de los datos se empleó el paquete estadístico SPSS para Windows (versión 17.0). Se han realizado estadísticos descriptivos de la muestra y de las puntuaciones en el pre y en el post. La fiabilidad se obtuvo mediante el alfa de Cronbach. Se han utilizado pruebas no paramétricas (test de Wilcoxon) para comparar las puntuaciones de actitudes y conocimientos pre y post. Para estudiar las relaciones entre importancia e implicación con los principios bioéticos en ambos momentos de la evaluación se han utilizado correlaciones bivariadas no paramétricas (Spearman).

\section{Resultados:}

\section{Características de la muestra}

La muestra total es de 63 profesionales de enfermería pertenecientes al servicio de nefrología (43 enfermeras y 20 auxiliares de enfermería) de un hospital de tercer nivel. Todos ellos respondieron al cuestionario de conocimientos, pero solo 23 (18 enfermeras y 5 auxiliares) lo hicieron al cuestionario de actitudes tanto en el pretest como en el postest.

\section{Resultados de la intervención}

\section{Comparaciones de medias pre / post intervención:}

a) Importancia principios bioéticos: No existen diferencias en la importancia que se atribuye a los principios bioéticos, siendo las medias muy elevadas tanto en el pre como en el postest.

b) Implicación Principios Bioéticos: Existen diferencias estadísticamente significativas en no maleficencia $(p=0,033)$ y en la puntuación global $(p=0,022)$, en la dirección de ser superiores una vez realizado el curso. 
c) Conocimientos: las diferencias encontradas -gestión emocional y comunicación difícil- son estadísticamente significativas $(p=0,00)$.
Esto indica que la asistencia al curso produjo efectos positivos en la adquisición de conocimientos. (Tabla 2).

\begin{tabular}{|c|c|c|c|}
\hline Importancia principios bioéticos & Media pre $(n=23)$ & Media post $(n=23)$ & Sig. de la diferencia \\
\hline Autonomía & $8,16(\mathrm{DT}=1,93)$ & $8,53(\mathrm{DT}=1,16)$ & n.s \\
\hline Justicia & $8,74(\mathrm{DT}=2,19)$ & $8,76(\mathrm{DT}=2,08)$ & n.s \\
\hline Beneficencia & $9,19(\mathrm{DT}=1,13)$ & $8,83(\mathrm{DT}=1,48)$ & n.s \\
\hline No Maleficencia & $9,47(\mathrm{DT}=0,88)$ & $9,52(\mathrm{DT}=0,82)$ & n.s \\
\hline Global & $8,88(\mathrm{DT}=1,23)$ & $8,89(\mathrm{DT}=1,27)$ & n.s \\
\hline Implicación principios bioéticos & Media pre $(n=23)$ & Media post $(n=23)$ & Sig. de la diferencia \\
\hline Autonomía & $7,58(\mathrm{DT}=2,66)$ & $8,21(\mathrm{DT}=1,71)$ & n.s \\
\hline Justicia & $7,71(\mathrm{DT}=2,79)$ & $8,11(\mathrm{DT}=1,64)$ & n.s \\
\hline Beneficencia & $8,33(\mathrm{DT}=2,11)$ & $8,76(\mathrm{DT}=1,59)$ & n.s \\
\hline No Maleficencia & $8,91(\mathrm{DT}=1,16)$ & $9,31(\mathrm{DT}=1,44)$ & $0,033^{*}$ \\
\hline Global & $8,17(\mathrm{DT}=2,01)$ & $8,58(\mathrm{DT}=1,30)$ & $0,022^{*}$ \\
\hline Conocimientos & Media pre $(n=63)$ & Media post $(n=63)$ & Sig. de la diferencia \\
\hline Manejo emocional & $1,96(\mathrm{DT}=1,23)$ & $3,59(\mathrm{DT}=1,15)$ & 0,000 ** \\
\hline Comunicación difícil & $5,04(\mathrm{DT}=2,04)$ & $6,52(\mathrm{DT}=1,87)$ & $0,000 * *$ \\
\hline
\end{tabular}

Tabla 2. Comparación de medias pre-post en las variables: Importancia principios bioéticos; Implicación principios bioéticos; Conocimientos

\begin{tabular}{|c|c|c|c|c|c|}
\hline PRE & $\begin{array}{c}\text { Implic. } \\
\text { Autonomía }\end{array}$ & Implic. Justicia & Implic. Benef. & $\begin{array}{c}\text { Implic. } \\
\text { No Maleficencia }\end{array}$ & Implic. Global \\
\hline Import. Autonomía & $0,62 * *$ & $0,63 * *$ & $0,54^{*}$ & $0,35^{*}$ & $0,60 * *$ \\
\hline Import. Justicia & 0,29 & $0,39 *$ & 0,12 & 0,18 & $0,31^{*}$ \\
\hline Import. Beneficencia & $0,55^{* *}$ & $0,58 * *$ & $0,51^{* *}$ & $0,65^{* *}$ & 0,68 ** \\
\hline Import. No Maleficencia & $0,54 * *$ & 0,47 ** & $0,51^{* *}$ & 0,68 ** & $0,63 * *$ \\
\hline Import. global & 0,60 ** & $0,64^{* *}$ & $0,40 * *$ & $0,49 * *$ & $0,65^{* *}$ \\
\hline POST & $\begin{array}{c}\text { Implic. } \\
\text { Autonomía }\end{array}$ & Implic. Justicia & Implic. Benef. & $\begin{array}{c}\text { Implic. } \\
\text { No Maleficencia }\end{array}$ & Implic. Global \\
\hline Import. Autonomía & 0,53 ** & $0,68 * *$ & 0,73 ** & $0,38^{*}$ & $0,38^{*}$ \\
\hline Import. Justicia & $0,56^{* *}$ & 0,66 ** & $0,62 * *$ & $0,46^{*}$ & $0,46^{*}$ \\
\hline Import. Beneficencia & $0,43^{*}$ & $0,62 * *$ & $0,69 * *$ & $0,52 * *$ & $0,52 * *$ \\
\hline Import. No Maleficencia & 0,56 ** & $0,59 * *$ & $0,60 * *$ & $0,91^{* *}$ & $0,91^{* *}$ \\
\hline Import. global & $0,66^{* *}$ & 0,74 ** & 0,76 ** & 0,60 ** & 0,60 ** \\
\hline \multicolumn{4}{|l|}{ Nota: ${ }^{*} p<0.05 ;{ }^{*} p<0.001$} & & \\
\hline
\end{tabular}

Tabla 3. Correlaciones Importancia-implicación principios éticos $(n=23)$ 
Relaciones entre la importancia y la implicación entre los principios bioeticos pre/post intervención

Tanto antes como después del taller la relación que se establece entre la importancia que se le da a los principios bioeticos y la implicación que se tiene con ellos en la práctica diaria es directa y significativa. (Tabla 3).

\section{Discusión:}

Los principales hallazgos de este estudio son que la participación de los profesionales de enfermería nefrológica en un curso de formación en gestión emocional, mejoró su implicación en el conjunto de los principios bioéticos y especialmente en la "no maleficencia", así como los conocimientos sobre manejo emocional y comunicación difícil. Este es el primer estudio llevado a cabo en nuestro país en una muestra de enfermería nefrológica sobre el impacto de un taller de formación en variables actitudinales y de conocimiento relacionadas con el ámbito de las emociones y la comunicación difícil con el paciente renal.

No se observan cambios en la importancia de los principios éticos (tienen valores altos de partida y se mantienen altos después de la intervención), pero sí en la implicación con los mismos, que sube significativamente en no maleficencia y en la puntuación global. Esto tiene sentido ya que uno de los ejes principales del curso es promover y trabajar la responsabilidad moral sobre la experiencia de sufrimiento, y esto queda reflejado en el principio de no maleficencia.

Tradicionalmente, este tipo de cursos están diseñados para dotar a las enfermeras de recursos y herramientas que les permitan hacer frente al manejo de situaciones de alto impacto emocional ${ }^{16}$. Los datos de nuestro estudio, apoyan la validez de la formación en habilidades de comunicación y manejo emocional en el personal de enfermería.

A pesar de haberse encontrado resultados interesantes, este estudio presenta ciertas limitaciones. Metodológicamente, el no haber incorporado una medida de seguimiento ni grupo control hace que sea difícil extraer conclusiones sobre la estabilidad de los cambios encontrados y sobre poder atribuir la causalidad de los resultados al efecto del programa. A pesar de ello, creemos que este tipo de acciones formativas son necesarias para dotar a las enfermeras de recursos comunicativos y de gestión emocional para su trabajo diario con los pacientes renales y sus familias.

Recibido: 1 Noviembre 2011

Revisado: 30 Noviembre 2011

Modificado: 30 Agosto 2012

Aceptado: 31 Agosto 2012

\section{Bibliografía}

1. Chochinov HM. Dignidad y la esencia de la medicina: El A, B, C y D del cuidado centrado en la dignidad. Medicina Paliativa, 2009; 16 (2): 95-99.

2. Bayés R. Medicina Paliativa: Psicología y Cuidados Paliativos. Medicina Paliativa, 2005; 12: 137-138.

3. Fallowfield L, Jenkins V, Farewell V, Saul J, Daffy A y Eves R. Efficacy of a Cancer Research UK communication skills training model for oncologists: A randomized controlled trial. The Lancet, 2002; 359: 650-656.

4. Barbero J. El derecho del paciente a la información: El arte de comunicar. Anales del Sistema Sanitario Navarro, 2006; 29 (3): 19-27.

5. Moss AH, Holley JL, Davidson SN, Dart RA, Germain MJ, Cohen L y Swartz, RD. Core Curriculum in nephrology: Palliative Care. American Journal of Kidney Diseases, 2004; 43 (1). 172-185.

6. Osterberg L. y Blaschke T. Adherence to medication. New England Journal of Medicine, 2005; 353 (5): 487-497.

7. Sarrias X, Bardón E y Vila ML. El paciente en pre-dialisis: toma de decisiones y libre elección terapéutica. Nefrología, 2008; 3: 119-122. 
8. Klerssy C, Callegari A., Martinelli V, Vizzardi V, Navino C, Malberti F, Tarchini R, Montagna G, Guastoni C, Bellazi R, Rampino T, David S, Barbieri C, Dal Canton A y Politi $P$ for the working group on burnout and dialysis. Burnout in health care providers of dialysis services in Northern Italy - a multicentre study. Nephrology, Dialysis and Transplantation, 2007; 22: 2283-2290.

9. Kruijver IP, Kerkstra A, Francke AL, Bensing JM y Van de Wiel HB. Evaluation of communication training programs in nursing care: a review of the literature. Patient, Education and Counselling, 2000; 39: 129-145.

10. Wilkinson S, Roberts A y Aldridge N. Nurse-patient communication in palliative care: an evaluation of a communication skills programme. Palliative Medicine, 1998; 12: 13-22.

11. Holley JL et al. The need of end-of-life care training in nephrology: national survey results of nephrology fellows. American Journal of Kidney Diseases, 2003; 42: 813-820.

12. García-Llana $H$, Barbero J, Remor E, Díaz-Sayas $L$, Rodríguez-Rey R, del Peso G y Selgas R. et al. Impacto de un curso interdisciplinar de Counselling y apoyo en la toma de decisiones a profesionales de un servicio de nefrología. American Nefrología, 2011; 31 (3): 322-330.

13. Barreto P, Arranz P y Molero M. Counselling, instrumento fundamental en la relación de ayuda. En: Martorell, M.C. y González R (eds). Entrevista y consejo psicológico (pp.83-104). Madrid: Síntesis, 1997.

14. Bartz R. Beyond the biopsychosocial model: New approaches to doctor-patient interactions. Journal of Family Practice, 1999; 48: 601-607.

15. Arranz, P. y Cancio, H. Counselling: habilidades de información y comunicación con el paciente oncológico. En: Gil, F. (Ed). Manual de Psico-oncología. Madrid: Nova Sidonia; 2000; 39-56.

16. Arranz P, Ulla S, del Rincón C y López-Fando T. Evaluation of a counselling training program for nursing staff. Patient, Education and Counselling, 2005; 56: 233-239. 